\title{
Light Activated Electrochemistry: Light Intensity and pH Dependence on Electrochemical Performance of Anthraquinone Derivatized Silicon
}

\author{
Ying Yang, ${ }^{\dagger}$ Simone Ciampi, ${ }^{*}{ }^{\ddagger}$ Moinul H. Choudhury, ${ }^{\dagger}$ and J. Justin Gooding ${ }^{*}{ }^{\dagger}$ \\ ${ }^{\dagger}$ School of Chemistry, The University of New South Wales, Sydney, NSW 2052, Australia \\ ${ }^{\ddagger}$ Intelligent Polymer Research Institute, University of Wollongong, Wollongong, NSW 2522, Australia
}

Supporting Information

ABSTRACT: We seek to understand how the thermodynamics and kinetics of anthraquinone-containing self-assembled monolayer on silicon electrodes are affected by two key experimental variables: the intensity of the light assisting the anthraquinone/anthrahydroquinone redox process and the local solution environment. The substrates are chemically passivated poorly doped p-type silicon electrodes. The study presents a strategy for the selective modulation of either the anodic or the cathodic process occurring at the interface. Cyclic voltammetry studies showed that unlike for a proton-coupled electron transfer process performed at metallic electrodes, for the redox reaction of the anthraquinone unit on a silicon electrode it becomes possible to (i) selectively facilitate only the oxidation process by increasing the electrolyte $\mathrm{pH}$ or (ii) at a given $\mathrm{pH}$ value to increase the illumination intensity to anodically shift the onset of the reduction step only but leave the oxidation process thermodynamic unchanged. A model concerning the proton coupled electron transfer

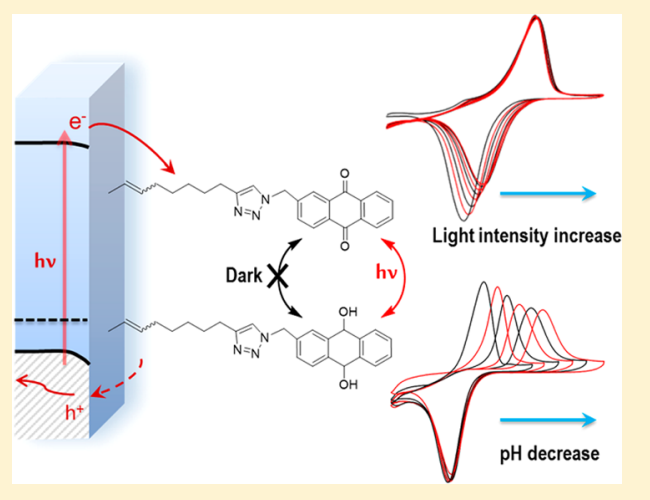
mechanism was proposed, where the electron transfer is the rate-determining step for the anthraquinone reduction while a deprotonation step is the rate-determining event for the anthrahydroquinone oxidation on poorly doped illuminated p-type silicon.

\section{INTRODUCTION}

Fundamental studies into semiconducting electrodes were pioneered in the 1970s and 1980s by researchers including Bard, Wrighton, and Lewis. ${ }^{1-8}$ These early works formed the basis for the use of silicon for the light-assisted reduction of $\mathrm{CO}_{2}{ }^{9}$, water splitting, ${ }^{10}$ and hydrogen generation. ${ }^{2,7,11}$ These technologies all rely on silicon being the photoresponsive material in which, upon the adsorption of light, electron-hole pairs are generated both in the field-free bulk and depletion region near the silicon/electrolyte interface (space charge). Carriers in the depletion layer can be separated by the electric field such that minority carriers are directed to the surface while the majority carriers are directed toward the bulk. ${ }^{12}$ Based on this principle, the obvious possibility of a two-dimensional confinement for the photoresponse led to the development of light addressable potentiometric sensors (LAPS) for biochemical monitoring ${ }^{13}$ and multianalyte detection. ${ }^{14-16}$ With regards to the former example, LAPS, the typical device consists of an electrolyte/insulator/semiconductor structure. An alternating photocurrent response is measured when the LAPS device is irradiated with a frequency-modulated light pointer at a specific position. The presence of charged species adjacent to the insulator surface influences the local characteristics of the space charge layer and hence the magnitude and phase of the alternating current. Thus, by measuring the amplitude or the phase of the photocurrent, a LAPS devices can realize detection of specific ions and biological species with the two-dimensional resolution down to $17 \pm 3 \mu \mathrm{m}$. ${ }^{17}$

The main limitation of LAPS is that only potentiometric sensing can be performed. This is because the insulating layer adds a significant kinetics barrier to electron transfer from and to the electrolyte. To broaden the scope of such an "addressing" concept in electroanalytical chemistry would require a method to allow faradaic electrochemistry to be performed specifically only at the site of illumination. This is what was achieved with the light activated electrochemistry concept we reported recently. ${ }^{18}$ With light activated electrochemistry, faradaic reactions can be performed wherever and whenever you want, with microscale resolution on an unstructured macroscopic monolithic silicon electrode simply by illuminating a discrete spot on that surface. To achieve lightactivated electrochemistry, three conditions must be fulfilled. First the semiconducting electrode must be biased in depletion, second the electrode must be modified such that it does not undergo photooxidation during electrochemistry but with a layer thin enough to allow efficient tunneling, and finally there

Received: December 10, 2015

Revised: January 4, 2016

Published: January 18, 2016 
must be sufficient electronic coupling with the redox active species as found when it is covalently bound to the surfacemodifying layer. In our preliminary study, ${ }^{18}$ we showed that these conditions could be fulfilled on poorly doped n-type "oxide-free" silicon electrodes that were modified with a selfassembled monolayer of 1,8-nonadiyne, to which a ferrocene unit was subsequently attached. This idea was shown to be applicable to writing electrochemical information to a surface, via the electrodeposition of polypyrrole, and to read electrochemical information from a discrete area of a macroscopic surface in terms of DNA hybridization sensing.

The conditions discussed above exist because the core principle of light-activated electrochemistry is that there are insufficient carriers for the electrochemistry to proceed in the dark, and irradiation causes photoexcitation of electrons (for a p-type silicon) from the valence band to the conduction band. There is a concomitant increase in the number of the minority carriers such that charge transfer can then proceed at an appreciable rate, and faradaic processes are observed. To further understand the scope of light-activated electrochemistry, however, it is important to examine the impact of (i) the light intensity on the faradaic process and (ii) with redox reactions that are environmentally sensitive, such as for protoncoupled electron transfer reactions, the interplay between photoeffects, and electrolyte composition on the electrochemistry. These effects will be investigated using nonadiyne modified p-type electrodes functionalized with anthraquinone via $\mathrm{Cu}(\mathrm{I})$-catalyzed alkyne-azide cycloaddition reaction ("click" or CuAAC). ${ }^{19-21}$ In this way, if a poorly doped ptype silicon electrode is utilized, it will only be able to sustain electron transfer to the anthraquinone at an appreciable rate when illuminated.

As a typical proton-coupled electron transfer process, the redox reaction of quinones has been studied extensively, both experimentally and theoretically. ${ }^{22-28}$ Laviron $^{22}$ and Finklea $^{23,24}$ proposed the nine-number square scheme on proton-coupled electron transfer reactions to account for a number of experimental observations. The stepwise mechanism of proton-coupled electron transfer is largely accepted. However, there are some recent experimental results obtained on proton coupled electron transfer reactions that do not fit with the stepwise mechanism of the square scheme; ${ }^{25,26}$ hence the possibility of concerted electron and proton transfer was introduced. $^{25,27}$ The exact proton-coupled electron transfer mechanism for anthraquinone redox process is therefore still a source of debate; ${ }^{27}$ however, there is general agreement on both thermodynamics and the kinetics being $\mathrm{pH}$ dependent. $^{23,28-31}$ The redox reaction of quinone and phenols derivatives do follow a shift in potential according to the Nernst equation, ${ }^{28,29,32}$ and the magnitude of the redox potential shift versus $\mathrm{pH}$ can be used to extract system parameters such as the number of electrons involved in the redox reaction. For quinones two electrons and two protons were involved in the redox reaction and slopes closed to -59.2 $\mathrm{mV} \mathrm{pH}^{-1}$ for equilibrium redox potential versus $\mathrm{pH}$ plots were observed. $^{28,31}$

Covalent attachment of on electrodes to form self-assembled monolayers allows controlling the local environment at the site of the redox reaction. There have been several studies on anthraquinone-terminated self-assembled monolayers on gold, glass carbon, and nickel electrodes. ${ }^{29,30,33-35}$ However, the electrochemistry for anthraquinone-terminated self-assembled monolayers at semiconducting silicon in aqueous environment is still unexplored. Therefore, the purpose of this work is to modify anthraquinone-terminated self-assembled monolayers on silicon substrate and to determine the effects of $\mathrm{pH}$ and light intensity on the thermodynamics and kinetics of anthraquinone redox reactions. The anodic and cathodic peak variations of anthraquinone-terminated silicon at different $\mathrm{pH}$ values as a function of light intensity were investigated by cyclic voltammetry. The rate constants for the electron transfer as well as the changes in space-charge characteristics with variation in illumination intensity were studied by electrochemical impedance spectroscopy.

\section{EXPERIMENTAL METHODS}

2.1. Chemicals and Materials. Unless otherwise specified, all chemicals are of analytical grade and used as received. Solvents used for the silicon cleaning steps were redistilled prior to use. Hydrogen peroxide (Sigma-Aldrich, $30 \mathrm{wt} \%$ in water), hydrofluoric acid (Riedel-Haën, $48 \mathrm{wt} \%$ in water), and sulfuric acid (J.T. Baker, 96\%) were of semiconductor grade. 1,8-Nonadiyne (Sigma-Aldrich, 98\%) was redistilled from sodium borohydride (Sigma-Aldrich, $\geq 99 \%$ ) under reduced pressure $\left(65-70{ }^{\circ} \mathrm{C}, 25-30\right.$ Torr $)$ and stored under a dry argon atmosphere prior to use. The redox species 2(azidomethyl)anthracene-9,10-dione was synthesized in one step from 2-(bromomethyl)anthracene-9,10-dione. Silicon substrates were prime grade, single-side polished, 100-oriented $\left(\langle 100\rangle \pm 0.9^{\circ}\right)$, $\mathrm{p}$ type (boron), with nominal resistivity of $0.007-0.009$ and $10-20 \Omega \mathrm{cm}$ and are referred to as highly and poorly doped, respectively. The poorly doped silicon were 500 $\pm 25 \mu \mathrm{m}$ thick and obtained from Virginia Semiconductors, Inc. (Fredericksburg, VA). The highly doped silicon were $525 \pm 25$ $\mu \mathrm{m}$ thick and were purchased from Siltronix, S.A.S. (Archamps, France).

2.2. X-ray Photoelectron Spectroscopy. X-ray photoelectron spectra were obtained on an ESCALAB 220iXL spectrometer fitted with a monochromatic $\mathrm{Al} \mathrm{K} \alpha$ source $(1486.6 \mathrm{eV})$, a hemispherical analyzer, and a six multichannel detector. The data were collected in normal emission at room temperature and a pressure below $10^{-9} \mathrm{mbar}$. The resolution of the spectrometer was $\sim 0.6 \mathrm{eV}$ as measured from the $\mathrm{Ag} 3 \mathrm{~d}_{5 / 2}$ signal full width at half-maximum. All binding energies are corrected by applying a rigid shift to bring the $\mathrm{C}$ 1s signal to $285.0 \mathrm{eV}$. The Thermo Avantage software was used for the analysis of XPS data. The atomic compositions were corrected for atomic sensitivities and measured from high-resolution scans. Atomic sensitivity factors are often instrument-sensitive, and the atomic sensitivities obtained from the Avance software interfaced with the spectrometer were 0.817 for Si 2p, 1.000 for C 1s, and 1.800 for $\mathrm{N} 1 \mathrm{~s}$.

2.3. Surface Modification Procedures. 2.3.1. Passivation of Silicon(100) with Monolayers of 1,8-Nonadiyne. The assembly of monolayers of 1,8-nonadiyne was performed on silicon following a previously reported procedure. ${ }^{20,36}$ As shown in Scheme 1, a silicon wafer (ca. $10 \times 30 \mathrm{~mm}$ ) was rinsed with dichloromethane, dried under a stream of argon, and then cleaned in hot piranha solution $\left(100{ }^{\circ} \mathrm{C}\right.$, a $1: 3(\mathrm{v} / \mathrm{v})$ mixture of $30 \%$ aqueous hydrogen peroxide to concentrated sulfuric acid for $30 \mathrm{~min}$. (Caution: piranha solution is dangerous when hot and explosive when mixing with organic compounds.) The sample was subsequently rinsed with water and etched with $2.5 \%$ aqueous hydrogen fluoride solution for $90 \mathrm{~s}$, before being transferred to a neat 1,8 nonadiyne sample which had been degassed through a 
Scheme 1. Surface Modification Processes: (a) Single-Side Polished Silicon(100) Wafers Were Immersed in $2.5 \% \mathrm{HF}$ for $90 \mathrm{~s}$ to Remove the Silicon Oxidation Layer; (b) Obtained Hydride-Terminated Surface Was Reacted with a Neat Sample of 1,8-Nonadiyne via Hydrosilylation Process; (c) Copper-Catalyzed Azide-Alkyne Cycloaddition (CuAAC) Reaction of an Azide-Tagged Anthraquinone Derivative on Acetylene-Terminated Silicon(100) Surface

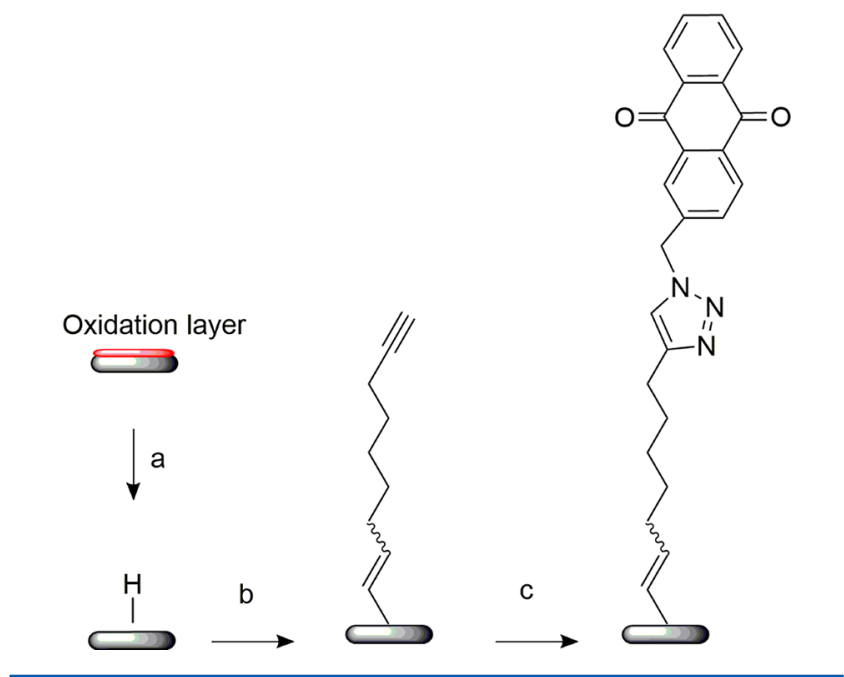

minimum of five freezing-pump-thawing cycles. The sample was kept under an argon atmosphere while the reaction flask was immersed for a $3 \mathrm{~h}$ period in an oil bath set to $165^{\circ} \mathrm{C}$. The reaction vessel was allowed to cool to room temperature, and the passivated silicon wafer was rinsed several times with dichloromethane, ethanol, immersed in dichloromethane at 4 ${ }^{\circ} \mathrm{C}$ for $2 \mathrm{~h}$, blown dry under argon, and stored under argon for further processing or analysis.

2.3.2. "CuAAC" Attachment of Redox-Active 2-(Azidomethyl)anthracene-9,10-dione. Anthraquinone was attached on alkyne-functionalized silicon(100) samples through the CuAAC reactions (Scheme 1). ${ }^{19,37}$ The passivated silicon sample was placed in a dimethyl sulfoxide solution containing 2 $\mathrm{mM}$ 2-(azidomethyl)anthracene-9,10-dione, $9.76 \mathrm{mM}$ copper(I) bromide, and $20.6 \mathrm{mM}$ tetramethylethylenediamine. Reactions were carried out at room temperature for $30 \mathrm{~min}$ without excluding air from the reaction environment. The prepared silicon samples were rinsed consecutively with copious amounts of water, ethanol, and dichloromethane, blown dry under argon, and stored under argon before being analyzed.

2.4. Electrochemical Measurements. All electrochemical experiments were performed in a PTFE three-electrode singlecompartment cell with the modified silicon electrode as the working electrode, a platinum mesh as the counter electrode, and $\mathrm{Ag} \mid \mathrm{AgCl}$ in $1 \mathrm{M} \mathrm{KCl}$ as the reference electrode. All potentials are reported versus the reference electrode. A rectilinear cross-section Viton gasket defined the geometric area of the working electrode to $26 \mathrm{~mm}^{2}$. The bulk of the backside of the silicon sample was exposed with emery paper and rubbed with gallium indium eutectic. A copper electrode was pressed against the sample backside, and it served as the ohmic contact. The cell was enclosed in a grounded Faraday cage during all measurements. The cyclic voltammetry were performed using a BAS 100B electrochemical analyzer (Bioanalytical Systems, Inc., West Lafayette, IN). The apparent rate constant for electron transfer $\left(k_{\mathrm{et}}{ }^{\prime}\right)$ was calculated from the simplified circuit ${ }^{38}$ of electrochemical impedance spectroscopy; the values were in reasonable agreement with the values from Laviron method (Table S1, Supporting Information). ${ }^{39}$ Flat band potential values were calculated from the extrapolation to $\mathrm{C}_{\mathrm{sc}}{ }^{-2}=0$ of Mott-Schottky $(M-S)$ plots $\left(C_{\mathrm{sc}}{ }^{-2}\right.$ vs $\left.E\right)$. Electrochemical impedance spectroscopy under different light intensity was recorded using Solartron model SI 1287 with a Solartron impedance/gain-phase analyzer. The half-wave potential $\left(E_{1 / 2}\right)$ was set as dc bias potential, and the ac amplitude was $10 \mathrm{mV}$. Experiments were performed in Britton Robinson (B\&R) buffer ${ }^{40}$ with a $\mathrm{pH}$ range 5.06-10.09. All the buffer solutions were degassed prior to experiments by bubbling argon through them for $20 \mathrm{~min}$.

ACE Light Source (SCHOTT AG, Mainz, Germany) was employed to perform the top-side (i.e., electrolyte side) illumination for electrochemical experiments. The light intensities were determined using a 1918-R optical power meter (Newport, Inc., Bozeman, MT). All through the present work, the illumination was performed from the solution side of the electrode surface, henceforth referred to as top-side illumination.

\section{RESULTS}

3.1. Spectroscopic Characterization of Anthraquinone Assemblies on Silicon. The chemical passivation of the silicon surface has been extensively characterized, ${ }^{20,36,41,42}$ and representative XPS spectra for the alkyne-terminated poorly doped p-type silicon(100) surface are shown in Figure S1. In addition to the bands of $\mathrm{Si}$, signals are observed for $\mathrm{C}$ and $\mathrm{O}$. In high-resolution Si 2 p spectra, the spin-orbit split peaks for Si $2 \mathrm{p}_{3 / 2}$ and $\mathrm{Si} 2 \mathrm{p}_{1 / 2}$ are shown at 99.5 and $100.1 \mathrm{eV}$, respectively. Three C 1s features from $\mathrm{C} 1 \mathrm{~s}$ core level spectra at 283.77 (C$\mathrm{Si}), 285.0(\mathrm{C}-\mathrm{C})$, and $286.4 \mathrm{eV}(\mathrm{C}-\mathrm{O}-\mathrm{H})$ are present on 1,8 nonadiyne passivated surfaces as discussed previously. ${ }^{21,36,42}$

The further modification of the alkyne-terminated silicon electrode by attachment of anthraquinone derivative (Scheme 1) was spectroscopically monitored by XPS (Figure S2). The utilization of CuAAC reactions on alkyne-terminated silicon surface for grafting azide-tagged molecules has been detailed extensively in previous studies. ${ }^{14-16}$ Concurring with expectations, the high-resolution N 1s XPS spectra (Figure S2a) for anthraquinone modified silicon are fitted to two distinct peaks having binding energies of 401.7 and $400.3 \mathrm{eV}$ and a 1:2 ratio of the integrated area, which are corresponding to the triazole $\mathrm{N}-$ $\mathrm{N}=\mathrm{N}$ and $\mathrm{C}-\mathrm{N}-\mathrm{N}$, respectively. ${ }^{19,20,37}$ The presence of a triazole group is evidence for the success for alkyne-azide cycloaddition reaction. The success of the reaction was further verified by high-resolution C 1s XPS narrow scans (Figure S2c). The low-energy peak centered at 283.8 was attributed to $\mathrm{C}-\mathrm{Si}$, while the $\mathrm{C} 1 \mathrm{~s}$ peaks with binding energy of 285.0 and $284.5 \mathrm{eV}$ were ascribed to carbon-bonded carbon atoms in aliphatic and aromatic configuration, respectively. The two high-energy peaks centered at $288.0 \mathrm{eV}(\mathrm{C}=\mathrm{O}$ for carbonyl group) and $286.5 \mathrm{eV}(\mathrm{C}-\mathrm{N})$ are also consistent with the anthraquinone modification on silicon. ${ }^{42-45}$ Importantly, the lack of XPS emissions in the energy range 101-105 eV indicates there is no appreciable substrate oxidation upon the CuAAC reaction. ${ }^{46,47}$

3.2. Light and Dark Redox Behavior of Anthraquinone Derivatized p-Type Silicon Electrodes. The redox response of the anthraquinone-functionalized p-type silicon was first studied using cyclic voltammetry (Figure 1). As evident from 

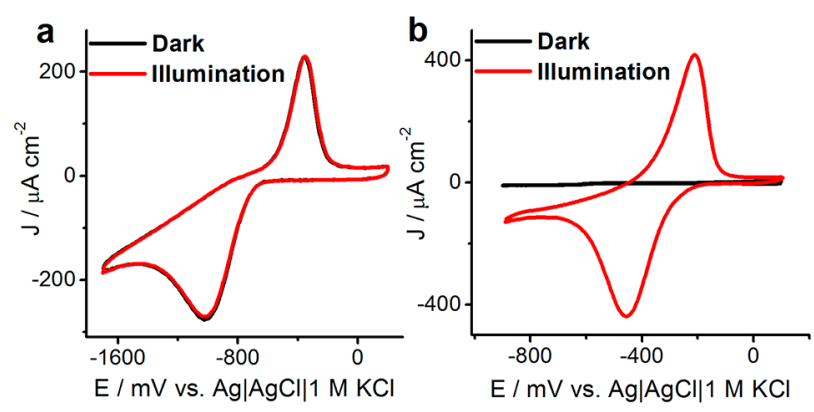

Figure 1. Cyclic voltammograms for anthraquinone-functionalized (a) highly (0.007-0.009 $\Omega \mathrm{cm}$ resistivity) and (b) poorly doped (10-20 $\Omega \mathrm{cm}$ resistivity) p-type silicon(100) in B\&R buffer $(\mathrm{pH}=10.06)$ in dark or under illumination. Scan rate was $2 \mathrm{~V} / \mathrm{s}$.

the cyclic voltammograms in aqueous electrolytes, the protonation of the radical anion collapses the anthraquinone reduction reactions to a single two-electron process. ${ }^{33}$ For highly doped p-type silicon there is no appreciable difference between voltammograms in the dark or under illumination (Figure 1a). Conversely, for poorly doped p-type silicon electrodes in the dark there is no evidence within the same potential window of any faradaic process, which accords well with the previous findings for poorly doped p-type silicon (Figure 1b). ${ }^{48,49}$ However, illumination of the electrode with light of energy greater than the band gap leads to dramatic changes in the voltammetry. In the dark, electrons are in depletion at the bias required for the reduction of anthraquinone to hydroquinone. Illumination generates electron-hole pairs and significantly increases the number of electrons in conduction band, which will be further driven to the silicon/electrolyte interface. ${ }^{12,50}$ As a consequence, there are sufficient carriers for electron transfer to proceed at anthraquinone-terminated silicon/electrolyte interface; hence, faradaic electrochemistry is observed. Compared with cyclic voltammograms of anthraquinone on metal electrodes ${ }^{29,51,52}$ the anodic and cathodic peak potentials $\left(E_{\mathrm{pa}}\right.$ and $\left.E_{\mathrm{pc}}\right)$ for anthraquinone on poorly doped $\mathrm{p}$-type silicon appear at more positive potentials. For example, the $E_{1 / 2}$ for anthraquinone derivative on gold electrode was found at $\mathrm{pH} 10$ approximately $-550 \mathrm{mV}$ vs AglAgCl $3 \mathrm{M} \mathrm{KCl}^{29}$ while on poorly doped p-type silicon, light can drive the anthraquinone electrochemical reaction at $\sim 220 \mathrm{mV}$ positive than the $E_{1 / 2}$ of the couple on gold electrode at the same $\mathrm{pH}$ value. From analysis of the area under the anodic wave, and considering a two-electron process, the surface coverage of anthraquinone moieties was estimated to be $(1.49 \pm 0.19) \times 10^{-10} \mathrm{~mol} \mathrm{~cm}^{-2}$, which is lower than the reported value (ca. $5 \times 10^{-10} \mathrm{~mol} \mathrm{~cm}{ }^{-2}$ ) for fully covered monolayers of hydroquinone on gold surface. ${ }^{53}$

3.3. Effects of Electrolyte pH on the Voltammetry of Anthraquinone Derivatized Poorly Doped p-Type Silicon. A representative set of cyclic voltammograms for anthraquinone modified p-type silicon were recorded in buffered electrolytes of $\mathrm{pH}$ values between 5.06 and 10.09 (Figure 2a and Figure S3a). Our data showed on anthraquinone modified highly doped p-type silicon that $E_{1 / 2}$ shifts with a slope of $-58.5 \mathrm{mV} \mathrm{pH}^{-1}$ (Figure S3b). In contrast, the $E_{1 / 2}$ on poorly doped p-type silicon shifts to more negative values by $-44.0 \mathrm{mV} \mathrm{pH}^{-1}$. The enlarged separation between the oxidation and reduction peaks with a decrease of $\mathrm{pH}$ implies the possible electrode kinetic changes. A quantification of the apparent rate constant for the electron transfer, $k_{\mathrm{et}}{ }^{\prime}$, was
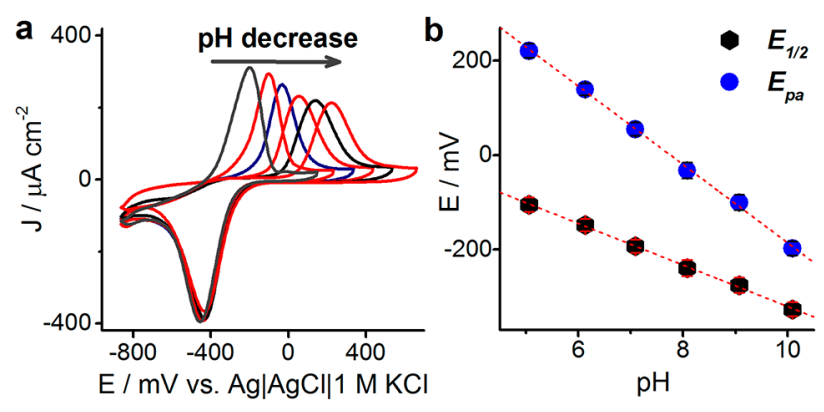

Figure 2. (a) Cyclic voltammetry of anthraquinone-modified poorly doped, p-type silicon at different $\mathrm{pH}$ values under illumination (light intensity $=94.2 \mathrm{~mW} \mathrm{~cm}^{-2}$, with scan rate $2 \mathrm{~V} / \mathrm{s}$ ). (b) Dependence of half-wave potential $\left(E_{1 / 2}\right)$ and anodic peak potential $\left(E_{\mathrm{pa}}\right)$ values with $\mathrm{pH}$. Dashed lines in (b) are plots of the fitted linear equations, $E_{1 / 2}=$ $119.2-44.0 \mathrm{pH}$ and $E_{\mathrm{pa}}=644.3-83.0 \mathrm{pH}$.

measured by electrochemical impedance spectroscopy (EIS). The $k_{\mathrm{et}}{ }^{\prime}$ on anthraquinone-terminated poorly doped p-type silicon was found to decrease from $4.4 \mathrm{~s}^{-1}$ at $\mathrm{pH} 10.09$ to 0.02 $\mathrm{s}^{-1}$ at $\mathrm{pH} 5.06$, while the $k_{\mathrm{et}}{ }^{\prime}$ on anthraquinone-terminated highly doped p-type silicon decreased from 1.4 to $0.01 \mathrm{~s}^{-1}$ (Table S1 and Figure S5a). The trend line for logarithmic $k_{\mathrm{et}}{ }^{\prime}$ versus $\mathrm{pH}$ was consistent with the previous studies. ${ }^{29,54,55}$ Uniquely, the influence of $\mathrm{pH}$ on $E_{\mathrm{pa}}$ and $E_{\mathrm{pc}}$ for anthraquinone modified silicon is quite different. The dependency of $E_{\mathrm{pc}}$ on the $\mathrm{pH}$ was linear with a very small slope of ca. $-5.2 \mathrm{mV} \mathrm{pH}^{-1}$, about 15 times smaller than dependency of $E_{\mathrm{pa}}$ on the $\mathrm{pH}$, which was $-83.1 \mathrm{mV} \mathrm{pH}^{-1}$. In previous studies, the influence of $\mathrm{pH}$ on the thermodynamics of anthraquinone-terminated selfassembled monolayers on gold electrodes followed the Nernst equation, ${ }^{29,53-55}$ with a shift in apparent formal potential of $-59.2 \mathrm{mV} \mathrm{pH}^{-1}$ between $\mathrm{pH} 1$ and $13 .^{31,53,55}$ The electrochemical reaction was shown to be a two-electron and twoproton reaction. Our data at anthraquinone modified poorly doped p-type silicon electrodes showed a $\mathrm{pH}$ dependence of $E_{1 / 2}$ is significantly smaller. This is because of the near negligible $\mathrm{pH}$ dependence of the cathodic $\left(-5.2 \mathrm{mV} \mathrm{pH}^{-1}\right)$ peak even though the $\mathrm{pH}$ dependence of the anodic peak is much greater than expected $\left(-83.1 \mathrm{mV} \mathrm{pH}^{-1}\right)$.

3.4. Light Intensity Dependence on the Voltammetry of Anthraquinone Derivatized Poorly Doped p-Type Silicon. To further explore the photoeffects on the redox reaction of anthraquinone moieties on poorly doped silicon, cyclic voltammetry was performed under variable light intensities (10.3, 16.7, 25.8, 38.2, 52.0, 69.6, 86.7, and 94.2 $\mathrm{mW} \mathrm{cm}^{-2}$ ) in B\&R buffer at a $\mathrm{pH}$ of 10.1 (Figure 3). Typical voltametric curves of light-driven anthraquinone redox processes are shown in Figure 3a. The increased light intensity drives the $E_{1 / 2}$ to more positive potential, with $E_{\mathrm{pa}}$ around -200 $\pm 4 \mathrm{mV}$ showing no obvious response to light intensity, while $E_{\mathrm{pc}}$ moved toward positive potential with the increase of light intensity. Figure $3 \mathrm{~b}$ shows light intensity dependence of $E_{\mathrm{pc}}$. The difference in $E_{\mathrm{pc}}$ was as much as $88.1 \pm 5.8 \mathrm{mV}$ as the light intensity was changed from 10.3 to $94.2 \mathrm{~mW} \mathrm{~cm}^{-2}$.

The electrode kinetics as a function of the light intensity was also studied, and it is found that the $k_{\text {et }}{ }^{\prime}$ for the electron transfer process increased with increasing light intensities (Table S1 and Figure S5b). Specifically, the experimental obtained $k_{\text {et }}{ }^{\prime}$ increased from $0.5 \mathrm{~s}^{-1}$ at a light intensity of $10.3 \mathrm{~mW} \mathrm{~cm}^{-2}$ to $4.4 \mathrm{~s}^{-1}$ at a light intensity of $94 \mathrm{~mW} \mathrm{~cm}^{-2}$. 

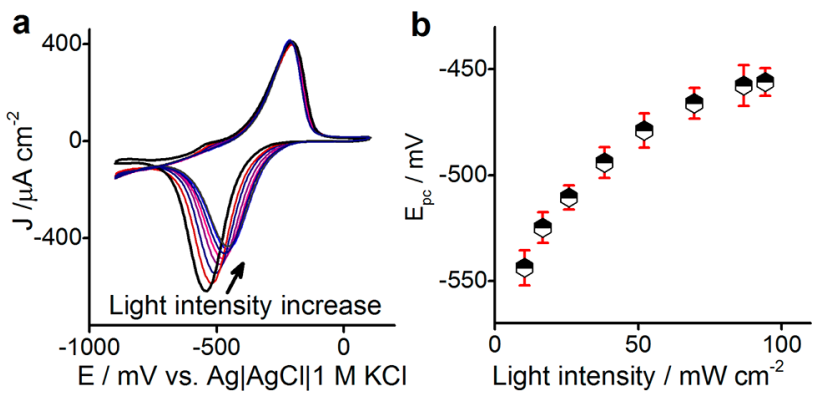

Figure 3. (a) Cyclic voltammetry of anthraquinone-terminated p-type silicon electrodes under different light intensities (10.3, 16.7, 25.8, 38.2, 52.0, 69.6, 86.7, and $94.2 \mathrm{~mW} \mathrm{~cm}^{-2}$ ) in B\&R buffer ( $\mathrm{pH} 10.1$ ). (b) Evolution of $E_{\mathrm{pc}}$ with light intensities. Illumination is operated on the whole surface from top side at the indicated intensity.

EIS experiments were performed under variable light intensity to investigate changes in the space charge layer as a result of the light and account for the apparent changes in the electron transfer kinetics. The Nyquist plots in Figure 4 exhibit

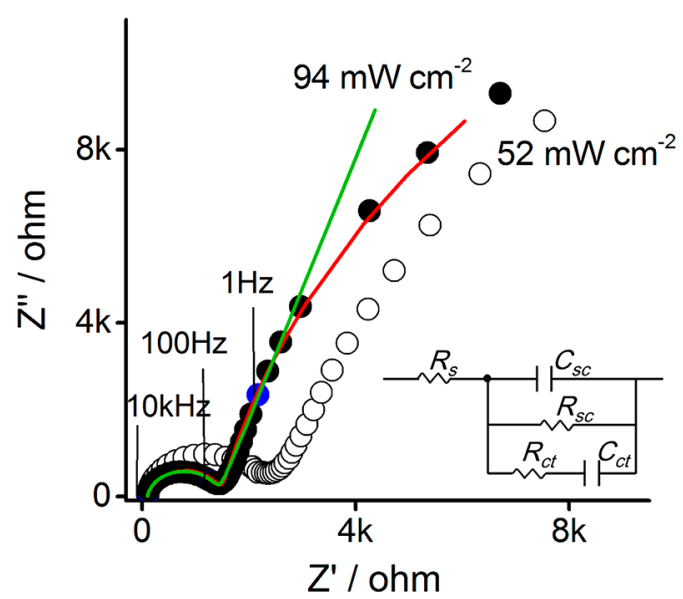

Figure 4. Nyquist plot for anthraquinone-terminated poorly doped ptype silicon at $\mathrm{pH} 10.06$ under indicated light intensity. Circles are data from $100 \mathrm{kHz}$ to $0.1 \mathrm{~Hz}$, and red solid line is the best fit to the equivalent circuit shown in the inset, with a $\chi^{2}$ statistics $\left(R^{2}\right)$ of 0.001 ; the green solid line is the best fit to the simplified equivalent circuit by removing $R_{\mathrm{so}}$ with $R^{2}$ of 0.01 .

a complete semicircle at the beginning and a second semicircle related to a slower process. The equivalent circuit used to refine the impedance data for the illuminated electrodes ${ }^{56,57}$ is shown in the inset. The semicircle in the high frequency region relates to the charge transfer process at the surface $\left(R_{\mathrm{ct}}\right.$ and $\left.C_{\mathrm{ct}}\right)$. The second semicircle arises from the space charge layer which is described by $R_{\mathrm{SC}}$ and $C_{\mathrm{SC}}$. The series resistance is $R_{\mathrm{s}}$. The Helmholtz layer was neglected in this case (infinite capacitance). The experimental $R_{\mathrm{SC}}$ and $C_{\mathrm{SC}}$ change with light intensity was shown in a logarithmic scale in Figure S6. With the increase of the illumination intensity there was an increase in $C_{\mathrm{sc}}$ and a slight decrease of $R_{\mathrm{sc}}$.

3.5. Flat Band Potential Measurement by MottSchottky Plots. The flat band potential $\left(E_{\mathrm{fb}}\right)$ is an experimental parameter that can help defining the silicon band energy positions with respect to the potential of electroactive species in an electrolyte. ${ }^{58}$ In the context of light activated electrochemistry, the knowledge of the value of the $E_{\mathrm{fb}}$ is vital as it indicates over which bias range the electrode is expected to be in depletion - a key prerequisite for observing photoelectrochemical effects such as the light "switch on" of a faradaic process in a bias window where the depleted dark interface behaves like an ideally polarizable system (i.e., infinite resistance). As previously described, ${ }^{59-61}$ Mott-Schottky plots can be employed to determine $E_{\mathrm{fb}}$ from the relationship between the applied potential $V$ and the space charge capacitance $C_{\mathrm{sc}}$ using the Mott-Schottky equation ${ }^{59-61}$

$$
C_{\mathrm{sc}}^{-2}=\frac{2}{\varepsilon \varepsilon_{0} q N A^{2}}\left(E-E_{\mathrm{fb}}-\frac{k T}{q}\right)
$$

where $\varepsilon$ is the dielectric constant for silicon, $\varepsilon_{0}$ the vacuum permittivity, $q$ the charge of the electron, $A$ the electrode surface area, $N$ the doping density of silicon, $E$ the applied potential, and $E_{\mathrm{fb}}$ the flat band potential, and at room temperature $k T / q$ is around $25 \mathrm{mV}$.

Figure S4 shows Mott-Schottky plots for the anthraquinone-terminated poorly doped $\mathrm{p}$-type silicon at different $\mathrm{pH}$ values, from 5.06 to 10.09 . In Mott-Schottky plots, linear regions were observed between -300 and $-600 \mathrm{mV}$. The intersection point of the potential and linear $C^{-2} A^{4}$ potential curve gives a flat band potential, which in this case moved negatively with the increase of $\mathrm{pH}$ values, with a slope -22.5 $\mathrm{mV} \mathrm{pH}^{-1}$. The $E_{\mathrm{fb}}$ dependent on $\mathrm{pH}$, which is also band edge shift with $\mathrm{pH}$, reflects a minor amount of oxidation of silicon, below the resolution of the XPS, when contacted with aqueous. The $\mathrm{pH}$ sensitivity was consistent with previous $\mathrm{pH}$ response of $\mathrm{CH}_{2} \mathrm{CHCH}_{2}$-Si-terminated silicon $\left(-28 \mathrm{mV} \mathrm{pH}^{-1}\right)$ by Lewis and others. ${ }^{62}$ The carrier density $\left((6.2 \pm 0.6) \times 10^{14} \mathrm{~cm}^{-3}\right)$ calculated from Mott-Schottky plots is consistent with the nominal resistivity values $\left((6-12) \times 10^{14} \mathrm{~cm}^{-3}\right) .63$

\section{DISCUSSION}

Here the p-type silicon was functionalized using the hydrosilylation reaction to form alkyl monolayer that protects the silicon against oxidation, followed by attaching an anthraquinone derivatives using a "click" reaction. ${ }^{20,36}$ Detailed characterization of XPS demonstrated that the anthraquinone moieties were successfully attached on silicon. As oxidation of the silicon surface results in an insulating layer, a modifying layer that prevents oxidation of the silicon in aqueous solution is of the major importance for developing silicon-based electrodes. ${ }^{36,42}$ The absence of silicon oxidation peaks in XPS spectra after the formation of the redox active self-assembled monolayer shows that this chemistry gives stable surfaces that will allow electrochemistry to be performed in the aqueous media without changes in the electrochemical performance of the electrode. $^{43}$

Highly doped silicon can be considered as having an impurity band which overlaps with the conduction or valence band. The overlap of the two bands results in free carriers such that the highly doped silicon acts more like a metal than as a semiconductor. Therefore, voltammograms of anthraquinone monolayers on highly doped silicon are very similar of those obtained on metallic electrodes. However, no redox behavior was observed at poorly doped silicon in the dark. Electrochemistry could be activated by illumination though. For poorly doped p-type silicon, the number of electrons in the conduction band is insufficient for the reduction of anthraquinone in the dark, and hence the faradaic processes were not observed in the cyclic voltammograms. ${ }^{3,7,12}$ However, electron-hole pairs generated inside the silicon after adsorption of light, and thus 
an appreciable increase of electrons in the conduction band, activate the reduction to proceed, and hence the whole redox process of anthraquinone is observed. $E_{1 / 2}$ on poorly doped ptype silicon was altered to a more positive potential compared with the $E_{1 / 2}$ of anthraquinone on metal electrodes, ${ }^{29,51,52}$ which reflects the ability to reduce anthraquinone contrathermodynamically by illumination. These results are consistent with the previous studies for the photoelectrochemical behavior of p-type silicon. ${ }^{2,3,7}$

Generally, with a $\mathrm{pH}$ increase, the $E_{1 / 2}$ of anthraquinone moieties on metallic electrode will decrease according to the Nernst equation in the referred $\mathrm{pH}$ range (with a value around $-59.2 \mathrm{mV}$ per $\mathrm{pH}$ under standard conditions). ${ }^{29}$ In the present work, the overall change of $E_{1 / 2}$ of anthraquinone on highly doped p-type silicon showed similar $\mathrm{pH}$ dependency with metallic electrodes. However, the anthraquinone-terminated poorly doped p-type silicon showed unexpected $\mathrm{pH}$ dependence on the redox behavior compared with the metal electrodes. The $E_{1 / 2}$ versus $\mathrm{pH}$ of anthraquinone for poorly doped p-type silicon was $-44.0 \mathrm{mV} \mathrm{pH}^{-1}$. Additionally, the $\mathrm{pH}$ dependences on $E_{\mathrm{pa}}$ and $E_{\mathrm{pc}}$ of anthraquinone on silicon electrode were quite different. The $\mathrm{pH}$ altered $E_{\mathrm{pa}}$ to a greater extent, while the linear dependency of $E_{\mathrm{pc}}$ on the $\mathrm{pH}$ value was very small.

The oxidation of hydroanthraquinone to anthraquinone involves loss of both electrons and protons. Together with the results from the Mott-Schottky plots, when the bias potential is more positive than flat band potentials, the silicon tends to be in accumulation. The oxidation of hydroanthraquinone could be considered as the injection of holes (valence band process) from the silicon. For p-type silicon electrode, the holes are in considerable excess of the electrons and are sufficient for the oxidation reaction to proceed. Hence, the deprotonation is probably the rate-limiting step. Supporting this notion is the positive shift in oxidation potential with a decrease in $\mathrm{pH}$, which indicates the greater the amount of protons in the solution environment around the anthraquinone, the more energy that is required for the oxidation to proceed. Hence, $\mathrm{pH}$ alters $E_{\mathrm{pa}}$ to a greater extent. According to our previous work of the volumetric behavior on anthraquinone-terminated $\mathrm{Au}(111)$, there was a linear relationship between $E_{\mathrm{pa}}$ and $\mathrm{pH}$ and the slope of dependency of $E_{\mathrm{pa}}$ on the $\mathrm{pH}$ was $-83.3 \mathrm{mV} \mathrm{pH}^{-1}$. The very similar dependency of $E_{\mathrm{pa}}$ with $\mathrm{pH}$ both for anthraquinone on gold electrode ${ }^{29}$ and silicon electrodes supports the hypothesis that the deprotonation was the rate determine step for the oxidation of anthraquinone on silicon.

In contrast, the onset of the reduction process, $E_{\mathrm{pc}}$ was insensitive to $\mathrm{pH}$, hence suggesting the rate-determining step to be related to electron transfer rather than proton transfer. The reduction process of anthraquinone happens when the silicon is under depletion. The minority carrier (electrons) transfer has significant influence and $\mathrm{pH}$ appears to have little impact on the position of the reduction peak. Overall, the position of $E_{\mathrm{pa}}$ and $E_{\mathrm{pc}}$ can be controlled by deprotonation and electron transfer separately; as a result, the observed $E_{1 / 2}$ of the cyclic voltammograms did not follow the Nernst equation as it does on metal electrodes.

The difference between the conduction band potential and the anthraquinone reduction potential can be considered as the driving force for electron flow from silicon to the interface for anthraquinone reduction. ${ }^{48,62}$ The $\mathrm{pH}$ sensitivity of conduction band potentials correlates with flat band potential, which shifts negatively with a $\mathrm{pH}$ increase. As electron transfer is the decisive factor for anthraquinone reduction on silicon, and the difference between the conduction band potential and the anthraquinone reduction potential was increased with $\mathrm{pH}$, electron transfer would be thus facilitated. Meanwhile, for the oxidation process, deprotonation of the anthrahydroquinone would become easier with increases in $\mathrm{pH}$ values. The measured overall $k_{\text {et }}{ }^{\prime}$ was found to increase with $\mathrm{pH}$, which was consistent with the prediction as well as the kinetics change with $\mathrm{pH}$ on highly doped p-type silicon or metal electrodes.

The consequence of the hypothesis above that electron transfer is the rate-limiting step for the reduction process while proton transfer dominated for the oxidation processs is that $E_{\mathrm{pc}}$ would be sensitive to light intensity while the $E_{\mathrm{pa}}$ will be insensitive to light intensity. This is in line with the experimental observations. The possible situations for the interface energetics in the present case are depicted in Scheme 2. The bands tend to be flatten with the light intensity

Scheme 2. Interface Energetics for Anthraquinone Derivatized Poorly Doped p-Type Silicon in Aqueous Electrolyte with Light Intensity Increase ${ }^{a}$

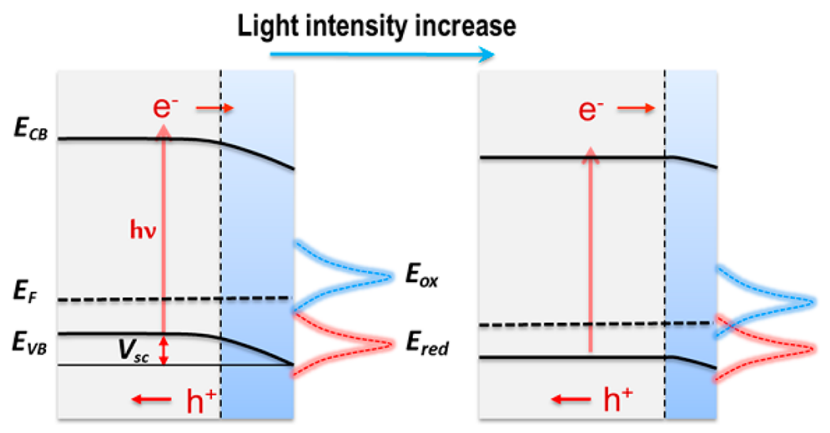

${ }^{a}$ The symbols $\mathrm{h}^{+}$and $\mathrm{e}^{-}$refer to holes and electrons, $E_{\mathrm{CB}}$ and $E_{\mathrm{VB}}$ stand for the conduction and valence band potentials of silicon, $E_{\mathrm{pc}}$ is the Fermi level of silicon, $E_{\mathrm{ox}}$ and $E_{\text {red }}$ are the reduction and oxidation peak potentials of anthraquinone, and $V_{\mathrm{sc}}$ is the potential drop across the space charger layer. All other terms have their usual meaning.

increase, ${ }^{64}$ and hence the Fermi level of silicon shifts, which leads to a change in electrode potential. This change was observed in Figure 3 where the reduction of anthraquinone became thermodynamically easier.

Our studies of the rate of electron transfer showed an increase in $k_{\mathrm{et}}{ }^{\prime}$ with increasing light intensity (Figure S5b). The dynamic processes at the silicon photoelectrode consists of minority carrier generation within the bulk, electron diffusion in the field free region, electron flow across the space charge layer, and charge transfer at the interface. ${ }^{65}$ Since with the increase of illumination intensity more electrons will be generated in the conduction band of silicon and the electron diffusion range increases as well, ${ }^{66,67}$ impedance spectroscopy was used to explore changes in the space charge layer, and interface charge transfer resistance, as a function of illumination intensity. From the results shown in Figure S6a, $C_{\mathrm{sc}}$ was calculated to increase with light intensity. According to previous work, the relationship between the potential distribution in depletion layer, and its width, can be expressed by the Poisson-Boltzmann equation. ${ }^{5,6,65,67-69}$ The potential drop across the depletion layer $\left(V_{\mathrm{sc}}\right.$ in Scheme 2) and its thickness would be smaller at higher light intensities. The $C_{\mathrm{sc}}$ shift is strong evidence for a decreased space charge layer width. At the same time, $R_{\mathrm{sc}}$ showed only a slight decrease with increased illumination 
intensity. With a marked increase of electron flux in the bulk semiconductor, and the decrease of space charge layer thickness, the charge transfer would thus be facilitated at anthraquinone-terminated silicon interface. This is in agreement with a concomitant decrease of $R_{\mathrm{ct}}$ and increase of $C_{\mathrm{ct}}$ (Figure S6b). As a consequence, the apparent kinetics for electron transfer with higher light intensity were faster (Figure S5). Note however the decrease of $R_{\mathrm{ct}}$ with increased light intensity becomes less significant at higher light intensities, suggesting the electrons at the interface are becoming sufficient. In concert, the measured $k_{\text {et }}{ }^{\prime}$ increased with light less significantly at higher light intensity.

\section{CONCLUSIONS}

In summary, an anthraquinone derivatized poorly doped p-type $\mathrm{Si}(100)$ system has been described, in which the $E_{1 / 2}$ of the redox species can be tuned either by changing the electrolyte $\mathrm{pH}$ or by varying the light intensity. The present work presents the significant $\mathrm{pH}$ dependency of the anodic process, leaving unaltered the cathodic event. Conversely, the position of the cathodic wave could be selectively tuned by changes in illumination intensity while the onset of the anodic event remains unaltered. The observation illustrates the oxidation and reduction state of anthraquinone relative to the energy bands of silicon. The oxidation process is a valence band process and holes transfer has negligible effects, while the reduction reaction relies on the electron transfer from the conduction band and the surface energetics has prominent influence. Both the anodic shift of reduction with respect to the silicon band level and increasing the intensity of illumination lead to easier electron transfer and hence facilitate the kinetics. The manipulation of silicon surfaces with anthraquinone and the possibility of tuning only the oxidation or reduction would serve to mediate the redox chemistry of solution species for further applications.

\section{ASSOCIATED CONTENT}

\section{S Supporting Information}

The Supporting Information is available free of charge on the ACS Publications website at DOI: 10.1021/acs.jpcc.5b12097.

Figures S1-S6 and Table S1 (PDF)

\section{AUTHOR INFORMATION}

\section{Corresponding Authors}

*E-mail sciampi@uow.edu.au (S.C.).

*E-mail justin.gooding@unsw.edu.au (J.J.G.).

\section{Notes}

The authors declare no competing financial interest.

\section{ACKNOWLEDGMENTS}

We thank Dr. Stephen Parker and Dr. Alexander H Soeriyadi for performing the XPS measurements. This research was supported by the Australian Research Council's Discovery Projects Funding Scheme (DP150103065) and the Centre of Excellence for Convergent Bio-Nano Science and Technology (CE140100036).

\section{REFERENCES}

(1) Bocarsly, A. B.; Walton, E. G.; Wrighton, M. S. Use of Chemically Derivatized N-Type Silicon Photoelectrodes in Aqueous Media. Photooxidation of Iodide, Hexacyanoiron(II), and Hexaammineruthenium(II) at Ferrocene-Derivatized Photoanodes. J. Am. Chem. Soc. 1980, 102, 3390-3398.
(2) Bookbinder, D. C.; Bruce, J. A.; Dominey, R. N.; Lewis, N. S.; Wrighton, M. S. Synthesis and Characterization of a Photosensitive Interface for Hydrogen Generation: Chemically Modified P-Type Semiconducting Silicon Photocathodes. Proc. Natl. Acad. Sci. U. S. A. 1980, 77, 6280-6284.

(3) Baglio, J. A.; Calabrese, G. S.; Harrison, D. J.; Kamieniecki, E.; Ricco, A. J.; Wrighton, M. S.; Zoski, G. D. Electrochemical Characterization of P-Type Semiconducting Tungsten Disulfide Photocathodes: Efficient Photoreduction Processes at Semiconductor/Liquid Electrolyte Interfaces. J. Am. Chem. Soc. 1983, 105, 22462256.

(4) Bolts, J. M.; Bocarsly, A. B.; Palazzotto, M. C.; Walton, E. G.; Lewis, N. S.; Wrighton, M. S. Chemically Derivatized N-Type Silicon Photoelectrodes. Stabilization to Surface Corrosion in Aqueous Electrolyte Solutions and Mediation of Oxidation Reactions by Surface-Attached Electroactive Ferrocene Reagents. J. Am. Chem. Soc. 1979, 101, 1378-1385.

(5) Bolts, J. M.; Wrighton, M. S. Chemically Derivatized N-Type Semiconducting Germanium Photoelectrodes. Persistent Attachment and Photoelectrochemical Activity of Ferrocene Derivatives. J. Am. Chem. Soc. 1978, 100, 5257-5262.

(6) Bolts, J. M.; Wrighton, M. S. Chemically Derivatized N-Type Semiconducting Gallium Arsenide Photoelectrodes. Thermodynamically Uphill Oxidation of Surface-Attached Ferrocene Centers. J. Am. Chem. Soc. 1979, 101, 6179-6184.

(7) Bookbinder, D. C.; Lewis, N. S.; Bradley, M. G.; Bocarsly, A. B.; Wrighton, M. S. Photoelectrochemical Reduction of N,N'-Dimethyl4,4'-Bipyridinium in Aqueous Media at P-Type Silicon: Sustained Photogeneration of a Species Capable of Evolving Hydrogen. J. Am. Chem. Soc. 1979, 101, 7721-7723.

(8) Bookbinder, D. C.; Bruce, J. A.; Dominey, R. N.; Lewis, N. S.; Wrighton, M. S. Synthesis and Characterization of a Photosensitive Interface for Hydrogen Generation: Chemically Modified P-Type Semiconducting Silicon Photocathodes. Proc. Natl. Acad. Sci. U. S. A. 1980, 77, 6280-6284.

(9) Kumar, B.; Smieja, J. M.; Kubiak, C. P. Photoreduction of Co2 on P-Type Silicon Using Re(Bipy-but) (Co)3cl: Photovoltages Exceeding $600 \mathrm{Mv}$ for the Selective Reduction of $\mathrm{Co} 2$ to Co. J. Phys. Chem. C 2010, 114, 14220-14223.

(10) Reece, S. Y.; Hamel, J. A.; Sung, K.; Jarvi, T. D.; Esswein, A. J.; Pijpers, J. J. H.; Nocera, D. G. Wireless Solar Water Splitting Using Silicon-Based Semiconductors and Earth-Abundant Catalysts. Science 2011, 334, 645-948.

(11) Abruna, H. D.; Bard, A. J. Semiconductor Electrodes. 40. Photoassisted Hydrogen Evolution at Poly(Benzyl Viologen)-Coated P-Type Silicon Electrodes. J. Am. Chem. Soc. 1981, 103, 6898-6901.

(12) Bard, A. J. Photoelectrochemistry. Science 1980, 207, 139-144.

(13) Hafeman, D. G.; Parce, J. W.; McConnell, H. M. LightAddressable Potentiometric Sensor for Biochemical Systems. Science 1988, 240, 1182-1185.

(14) Wagner, T.; Werner, C. F.; Miyamoto, K.-i.; Schoening, M. J.; Yoshinobu, T. Development and Characterisation of a Compact LightAddressable Potentiometric Sensor (Laps) Based on the Digital Light Processing (Dlp) Technology for Flexible Chemical Imaging. Sens. Actuators, B 2012, 170, 34-39.

(15) Wagner, T.; Molina, R.; Yoshinobu, T.; Kloock, J. P.; Biselli, M.; Canzoneri, M.; Schnitzler, T.; Schöning, M. J. Handheld MultiChannel Laps Device as a Transducer Platform for Possible Biological and Chemical Multi-Sensor Applications. Electrochim. Acta 2007, 53, $305-311$.

(16) Polsky, R.; Harper, J. C.; Wheeler, D. R.; Brozik, S. M. Multifunctional Electrode Arrays: Towards a Universal Detection Platform. Electroanalysis 2008, 20, 671-679.

(17) George, M.; Parak, W. J.; Gerhardt, I.; Moritz, W.; Kaesen, F.; Geiger, H.; Eisele, I.; Gaub, H. E. Investigation of the Spatial Resolution of the Light-Addressable Potentiometric Sensor. Sens. Actuators, A 2000, 86, 187-196. 
(18) Choudhury, M.; Ciampi, S.; Yang, Y.; Tavallaie, R.; Zhu, Y.; Zarei, L.; Goncales, V. R.; Gooding, J. J. Connecting Electrodes with Light: One Wire, Many Electrodes. Chem. Sci. 2015, 6, 6769-6776.

(19) Devadoss, A.; Chidsey, C. E. D. Azide-Modified Graphitic Surfaces for Covalent Attachment of Alkyne-Terminated Molecules by "Click" Chemistry. J. Am. Chem. Soc. 2007, 129, 5370-5371.

(20) Ciampi, S.; Boecking, T.; Kilian, K. A.; James, M.; Harper, J. B.; Gooding, J. J. Functionalization of Acetylene-Terminated Monolayers on $\mathrm{Si}(100)$ Surfaces: A Click Chemistry Approach. Langmuir 2007, 23, 9320-9329.

(21) Ciampi, S.; James, M.; Michaels, P.; Gooding, J. J. Tandem "Click" Reactions at Acetylene-Terminated $\mathrm{Si}(100)$ Monolayers. Langmuir 2011, 27, 6940-6949.

(22) Laviron, E. Electrochemical Reactions with Protonations at Equilibrium: Part Viii. The $2 \mathrm{E}, 2 \mathrm{~h}+$ Reaction (Nine-Member Square Scheme) for a Surface or for a Heterogeneous Reaction in the Absence of Disproportionation and Dimerization Reactions. J. Electroanal. Chem. Interfacial Electrochem. 1983, 146, 15-36.

(23) He, P.; Crooks, R. M.; Faulkner, L. R. Adsorption and Electrode Reactions of Disulfonated Anthraquinones at Mercury Electrodes. J. Phys. Chem. 1990, 94, 1135-1141.

(24) Finklea, H. O. Theory of Coupled Electron-Proton Transfer with Potential-Dependent Transfer Coefficients for Redox Couples Attached to Electrodes $\dagger$. J. Phys. Chem. B 2001, 105, 8685-8693.

(25) Wong, E. L. S.; Gooding, J. J. Electronic Detection of Target Nucleic Acids by a 2,6-Disulfonic Acid Anthraquinone Intercalator. Anal. Chem. 2003, 75, 3845-3852.

(26) Kullapere, M.; Tammeveski, K. Oxygen Electroreduction on Anthraquinone-Modified Nickel Electrodes in Alkaline Solution. Electrochem. Commun. 2007, 9, 1196-1201.

(27) Maia, G.; Maschion, F.; Tanimoto, S.; Vaik, K.; Mäeorg, U.; Tammeveski, K. J. Attachment of Anthraquinone Derivatives to Glassy Carbon and the Electrocatalytic Behavior of the Modified Electrodes toward Oxygen Reduction. J. Solid State Electrochem. 2007, 11, 14111420.

(28) Darwish, N.; Eggers, P. K.; Ciampi, S.; Tong, Y.; Ye, S.; PaddonRow, M. N.; Gooding, J. J. Probing the Effect of the Solution Environment around Redox-Active Moieties Using Rigid Anthraquinone Terminated Molecular Rulers. J. Am. Chem. Soc. 2012, 134, 18401-18409.

(29) Finklea, H. O.; Haddox, R. M. Coupled Electron/Proton Transfer of Galvinol Attached to Sams on Gold Electrodes. Phys. Chem. Chem. Phys. 2001, 3, 3431-3436.

(30) Lehmann, M. W.; Evans, D. H. Mechanism of the Electrochemical Reduction of 3,5-Di-Tert-Butyl-1,2-Benzoquinone. Evidence for a Concerted Electron and Proton Transfer Reaction Involving a Hydrogen-Bonded Complex as Reactant $\dagger$. J. Phys. Chem. B 2001, 105, 8877-8884.

(31) Costentin, C.; Robert, M.; Savéant, J.-M. Carboxylates as Proton-Accepting Groups in Concerted Proton-Electron Transfers. Electrochemistry of the 2,5-Dicarboxylate 1,4-Hydrobenzoquinone/ 2,5-Dicarboxy 1,4-Benzoquinone Couple. J. Am. Chem. Soc. 2006, 128, $8726-8727$.

(32) Costentin, C. Electrochemical Approach to the Mechanistic Study of Proton-Coupled Electron Transfer. Chem. Rev. 2008, 108, 2145-2179.

(33) Batchelor-McAuley, C.; Kozub, B. R.; Menshykau, D.; Compton, R. G. Voltammetric Responses of Surface-Bound and Solution-Phase Anthraquinone Moieties in the Presence of Unbuffered Aqueous Media. J. Phys. Chem. C 2011, 115, 714-718.

(34) Eggers, P. K.; Darwish, N.; Paddon-Row, M. N.; Gooding, J. J. Surface-Bound Molecular Rulers for Probing the Electrical Double Layer. J. Am. Chem. Soc. 2012, 134, 7539-7544.

(35) Sato, Y.; Fujita, M.; Mizutani, F.; Uosaki, K. Electrochemical Properties of the 2-Mercaptohydroquinone Monolayer on a Gold Electrode. Effect of Solution Ph, Adsorption Time and Concentration of the Modifying Solution. J. Electroanal. Chem. 1996, 409, 145-154.
(36) Costentin, C.; Robert, M.; Savéant, J.-M. Update 1 Of: Electrochemical Approach to the Mechanistic Study of ProtonCoupled Electron Transfer. Chem. Rev. 2010, 110, PR1-PR40.

(37) Darwish, N.; Eggers, P. K.; Da Silva, P.; Zhang, Y.; Tong, Y.; Ye, S.; Gooding, J. J.; Paddon-Row, M. N. Electroactive Self-Assembled Monolayers of Unique Geometric Structures by Using Rigid Norbornylogous Bridges. Chem. - Eur. J. 2012, 18, 283-292.

(38) Ciampi, S.; Eggers, P. K.; Le Saux, G.; James, M.; Harper, J. B.; Gooding, J. J. Silicon (100) Electrodes Resistant to Oxidation in Aqueous Solutions: An Unexpected Benefit of Surface Acetylene Moieties. Langmuir 2009, 25, 2530-2539.

(39) Rohde, R. D.; Agnew, H. D.; Yeo, W.-S.; Bailey, R. C.; Heath, J. R. A Non-Oxidative Approach toward Chemically and Electrochemically Functionalizing Si(111). J. Am. Chem. Soc. 2006, 128, 95189525.

(40) Creager, S. E.; Wooster, T. T. A New Way of Using Ac Voltammetry to Study Redox Kinetics in Electroactive Monolayers. Anal. Chem. 1998, 70, 4257-4263.

(41) Laviron, E. J. The Use of Linear Potential Sweep Voltammetry and of A.C. Voltammetry for the Study of the Surface Electrochemical Reaction of Strongly Adsorbed Systems and of Redox Modified Electrodes. J. Electroanal. Chem. Interfacial Electrochem. 1979, 100, 263-270.

(42) Britton, H. T. S.; Robinson, R. A. Cxcviii.-Universal Buffer Solutions and the Dissociation Constant of Veronal. J. Chem. Soc. 1931, 1456-1462.

(43) Ciampi, S.; James, M.; Darwish, N.; Luais, E.; Guan, B.; Harper, J. B.; Gooding, J. J. Oxidative Acetylenic Coupling Reactions as a Surface Chemistry Tool. Phys. Chem. Chem. Phys. 2011, 13, 1562415632.

(44) Ciampi, S.; Harper, J. B.; Gooding, J. J. Wet Chemical Routes to the Assembly of Organic Monolayers on Silicon Surfaces Via the Formation of Si-C Bonds: Surface Preparation, Passivation and Functionalization. Chem. Soc. Rev. 2010, 39, 2158-2183.

(45) Ciampi, S.; James, M.; Le Saux, G.; Gaus, K.; Gooding, J. J. Electrochemical "Switching" of Si(100) Modular Assemblies. J. Am. Chem. Soc. 2012, 134, 844-847.

(46) Ciampi, S.; Le Saux, G.; Harper, J. B.; Gooding, J. J. Optimization of Click Chemistry of Ferrocene Derivatives on Acetylene-Functionalized Silicon(100) Surfaces. Electroanalysis 2008, $20,1513-1519$

(47) Kilian, K. A.; Boecking, T.; Gaus, K.; Gal, M.; Gooding, J. J. Si-C Linked Oligo(Ethylene Glycol) Layers in Silicon-Based Photonic Crystals: Optimization for Implantable Optical Materials. Biomaterials 2007, 28, 3055-3062.

(48) Wallart, X.; Henry de Villeneuve, C.; Allongue, P. Truly Quantitative Xps Characterization of Organic Monolayers on Silicon: Study of Alkyl and Alkoxy Monolayers on H-Si(111). J. Am. Chem. Soc. 2005, 127, 7871-7878.

(49) Ciampi, S.; James, M.; Choudhury, M.; Darwish, N.; Gooding, J. J. The Detailed Characterization of Electrochemically Switchable Molecular Assemblies on Silicon Electrodes. Phys. Chem. Chem. Phys. 2013, 15, 9879-9890.

(50) Bocarsly, A. B.; Bookbinder, D. C.; Dominey, R. N.; Lewis, N. S.; Wrighton, M. S. Photoreduction at Illuminated P-Type Semiconducting Silicon Photoelectrodes. Evidence for Fermi Level Pinning. J. Am. Chem. Soc. 1980, 102, 3683-3688.

(51) Bookbinder, D. C.; Wrighton, M. S. Thermodynamically Uphill Reduction of a Surface-Confined N,N'-Dialkyl-4,4'-Bipyridinium Derivative on Illuminated P-Type Silicon Surfaces. J. Am. Chem. Soc. 1980, 102, 5123-5125.

(52) Lewis, N. S. Photoeffects at the Semiconductor/Liquid Interface. Annu. Rev. Mater. Sci. 1984, 14, 95-117.

(53) Hong, H.-G.; Park, W. Electrochemical Characteristics of Hydroquinone-Terminated Self-Assembled Monolayers on Gold. Langmuir 2001, 17, 2485-2492.

(54) Trammell, S. A.; Moore, M.; Schull, T. L.; Lebedev, N. Synthesis and Electrochemistry of Self-Assembled Monolayers Containing 
Quinone Derivatives with Varying Electronic Conjugation. J. Electroanal. Chem. 2009, 628, 125-133.

(55) Trammell, S. A.; Lowy, D. A.; Seferos, D. S.; Moore, M.; Bazan, G. C.; Lebedev, N. Heterogeneous Electron Transfer of QuinoneHydroquinone in Alkaline Solutions at Gold Electrode Surfaces: Comparison of Saturated and Unsaturated Bridges. J. Electroanal. Chem. 2007, 606, 33-38.

(56) Bohra, D.; Smith, W. A. Improved Charge Separation Via FeDoping of Copper Tungstate Photoanodes. Phys. Chem. Chem. Phys. 2015, 17, 9857-9866.

(57) Klahr, B.; Gimenez, S.; Fabregat-Santiago, F.; Hamann, T.; Bisquert, J. Water Oxidation at Hematite Photoelectrodes: The Role of Surface States. J. Am. Chem. Soc. 2012, 134, 4294-4302.

(58) Bard, A. J.; Bocarsly, A. B.; Fan, F. R. F.; Walton, E. G.; Wrighton, M. S. The Concept of Fermi Level Pinning at Semiconductor/Liquid Junctions. Consequences for Energy Conversion Efficiency and Selection of Useful Solution Redox Couples in Solar Devices. J. Am. Chem. Soc. 1980, 102, 3671-3677.

(59) Cooper, G.; Turner, J. A.; Nozik, A. J. Mott-Schottky Plots and Flat-Band Potentials for Single-Crystal Rutile Electrodes. J. Electrochem. Soc. 1982, 129, 1973-1977.

(60) De Gryse, R; Gomes, W. P.; Cardon, F.; Vennik, J. On the Interpretation of Mott-Schottky Plots Determined at Semiconductor/ Electrolyte Systems. J. Electrochem. Soc. 1975, 122, 711-712.

(61) Singh, P.; Singh, R.; Gale, R.; Rajeshwar, K.; DuBow, J. Surface Charge and Specific Ion Adsorption Effects in Photoelectrochemical Devices. J. Appl. Phys. 1980, 51, 6286-6291.

(62) Johansson, E.; Boettcher, S. W.; O'Leary, L. E.; Poletayev, A. D.; Maldonado, S.; Brunschwig, B. S.; Lewis, N. S. Control of the PhDependence of the Band Edges of $\mathrm{Si}(111)$ Surfaces Using Mixed Methyl/Allyl Monolayers. J. Phys. Chem. C 2011, 115, 8594-8601.

(63) Irvin, J. C. Resistivity of Bulk Silicon and of Diffused Layers in Silicon. Bell Syst. Technol. J. 1962, 41, 387-410.

(64) Bocarsly, A. B.; Walton, E. G.; Bradley, M. G.; Wrighton, M. S. Two-Electron Oxidations at Illuminated N-Type Semiconducting Silicon Electrodes: Use of Chemically Derivatized Photoelectrodes. J. Electroanal. Chem. Interfacial Electrochem. 1979, 100, 283-306.

(65) Krishnan, R. Fundamentals of Semiconductor Electrochemistry and Photoelectrochemistry; Wiley-VCH: Weinheim, 2007.

(66) Shimokawa, R.; Hayashi, Y. Effect of Illumination Wavelength and Intensity on Minority-Carrier Diffusion Length of Efg Silicon Ribbon Solar Cells. IEEE Trans. Electron Devices 1983, 30, 1770-1775.

(67) Marvin, D.; Halle, L. F. In Intensity Effects in Monitoring Carrier Lifetime Measurements. IEEE Photovoltaic Specialists Conference 1990, 1, 353-356.

(68) Pleskov, Y. V.; Gurevich, Y. Y. Semiconductor Photoelectrochemistry; Springer: New York, 2012.

(69) Kedem, N.; Brenner, T. M.; Kulbak, M.; Schaefer, N.; Levcenko, S.; Levine, I.; Abou-Ras, D.; Hodes, G.; Cahen, D. Light-Induced Increase of Electron Diffusion Length in a $\mathrm{P}-\mathrm{N}$ Junction Type Ch3nh3pbbr3 Perovskite Solar Cell. J. Phys. Chem. Lett. 2015, 6, 2469-2476. 